\title{
Density matrix renormalization group method and large-scale nuclear shell-model calculations
}

\author{
J. Dukelsky, ${ }^{1}$ S. Pittel, ${ }^{2}$ S. S. Dimitrova,${ }^{3}$ and M. V. Stoitsov ${ }^{3}$ \\ ${ }^{1}$ Instituto de Estructura de la Materia, Consejo Superior de Investigaciones Cientificas, Serrano 123, E-28006 Madrid, Spain \\ ${ }^{2}$ Bartol Research Institute, University of Delaware, Newark, Delaware 19716 \\ ${ }^{3}$ Institute for Nuclear Research and Nuclear Energy, Bulgarian Academy of Sciences, Sofia 1784, Bulgaria
}

(Received 5 February 2002; published 9 May 2002)

\begin{abstract}
The particle-hole density matrix renormalization group ( $p-h$ DMRG) method is discussed as a possible new approach to large-scale nuclear shell-model calculations. Following a general description of the method, we apply it to a class of problems involving many identical nucleons constrained to move in a single large $j$-shell and to interact via a pairing plus quadrupole interaction. A single-particle term that splits the shell into degenerate doublets is included so as to accommodate the physics of a Fermi surface in the problem. We apply the $p$ - $h$ DMRG method to this test problem for two $j$ values, one for which the shell model can be solved exactly and one for which the size of the Hamiltonian is much too large for exact treatment. In the former case, the method is able to reproduce the exact results for the ground state energy, the energies of low-lying excited states, and other observables with extreme precision. In the latter case, the results exhibit rapid exponential convergence, suggesting the great promise of this new methodology even for more realistic nuclear systems. We also compare the results of the test calculations with those from Hartree-Fock-Bogoliubov approximation and address several other questions about the $p-h$ DMRG method of relevance to its usefulness when treating more realistic nuclear systems.
\end{abstract}

DOI: 10.1103/PhysRevC.65.054319

PACS number(s): 21.60.Cs, 05.10.Cc

\section{INTRODUCTION}

The nuclear shell model [1] is one of the most powerful approaches for a microscopic description of nuclear properties. The low-energy structure of a given nucleus is described in this approach by assuming an inert doubly magic core and then diagonalizing the effective Hamiltonian within an active valence space consisting of at most a few major shells. Despite the enormous truncation inherent in this approach, the shell-model method as just described can still only be applied in very limited nuclear regimes, namely, for those nuclei with a sufficiently small number of active nucleons or a relatively low degeneracy of the valence shells that are retained. The largest calculations that have been reported to date are for the binding energies of nuclei in the $f p$ shell through ${ }^{64} \mathrm{Zn}[2]$.

For heavier nuclei or nuclei farther from closed shells, one is forced to make further truncations in order to reduce the number of shell-model configurations to a manageable size. The most promising approach now in use is to truncate on the basis of Monte Carlo sampling [3]. In this way, it has recently proven possible to extend the shell model beyond the $f p$ shell to describe the transition from spherical to deformed nuclei in the barium isotopes [4].

Another attractive possibility is provided by the density matrix renormalization group (DMRG), a method that was initially developed and applied in the framework of lowdimensional quantum lattice systems [5]. For a recent review, see Ref. [6]. A simplified version of this method was applied [7] to the two-level pairing model, showing its convergence properties. Subsequently, the method was extended to finite Fermi systems and applied to a pairing problem of relevance to ultrasmall superconducting grains [8]. This new methodology, which is referred to as the particle-hole (or $p-h$ ) DMRG, was recently applied to a first test problem of rel- evance to nuclear structure [9]. The application involved identical nucleons moving in a single large- $j$ shell under the influence of a pairing plus quadrupole interaction with an additional single-particle energy term that split the shell into doubly degenerate levels. Comparing with the results of exact diagonalization, it was shown that the method leads to extremely accurate results for the ground state and for lowlying excited states without ever requiring the diagonalization of very large matrices. Furthermore, even when the problem was not amenable to exact solution, the method was seen to exhibit rapid exponential convergence. All of this suggests that the DMRG method may indeed be a practical means of carrying out reliable large-scale shell-model calculations.

Before proceeding to realistic applications of the method, it is important to clarify its various ingredients and assumptions. Furthermore, it is critical to optimize how we apply the methodology for the subsequent more realistic and complex applications that will follow. With that in mind, we report here the results of a more thorough investigation of the recent application of the DMRG method to the one-orbit pairing plus quadrupole model. Several of the questions that were hinted at in Ref. [9] are now addressed, including the feasibility of applying the method to very much larger model spaces.

The outline of the paper is as follows. In Sec. II, we review the basic features of the DMRG method and provide a fairly comprehensive description of the theoretical ingredients required for its implementation. In Sec. III, we describe the one-shell model that we will be using for the first tests of the DMRG method in nuclear physics. In Sec. IV we present the results of these test calculations for a problem of ten identical nucleons restricted to move in a single $j=25 / 2$ shell. This problem can be solved exactly using the Lanczos algorithm, providing us with a good testing ground of the 
methodology including several variants not discussed in Ref. [9]. In Sec. V, we discuss the application of the method to a problem involving 40 nucleons in a $j=99 / 2$ orbit, a problem well beyond the limits of exact diagonalization. In Sec. VI, we summarize the principal conclusions of this work and outline some future directions for investigation.

\section{OVERVIEW OF THE $p$ - $h$ DMRG PROCEDURE}

\section{A. Qualitative description}

The basic idea of the $p-h$ DMRG method is to systematically take into account the physics of all single-particle levels. The $p-h$ nature of the method enters through a separation of the active single-particle levels into two sets that are separated by the Fermi surface; those above the Fermi surface are called the particle levels and those below are called the hole levels. The procedure begins by first taking into account the most important levels, namely, those that are nearest to the Fermi surface, and then gradually including the others in subsequent iterations. At each step of the procedure, a truncation is implemented both in the space of particle states and in the space of hole states, so as to optimally take into account the effect of the most important states for each of these two subspaces of the problem. The calculation is carried out as a function of the number of particle and hole states that are maintained after each iteration, with the assumption that these numbers are the same. This parameter, which we call $p$, is gradually increased and the results are plotted against it. Prior experience from other applications of the methodology suggests that the results converge exponentially with $p$. Thus, once we achieve changes with increasing $p$ that are acceptably small we terminate the calculation. Assuming that the convergence is rapid, the method permits us to achieve an accurate description of the low-lying states of the system without ever having to diagonalize the large matrices that would arise in the absence of the DMRG truncation strategy.

\section{B. Description of the method}

It is useful to now put the above qualitative remarks about the DMRG method into a more mathematical context.

The general problem that we wish to solve is a standard shell-model problem of $N_{\nu}$ neutrons and $N_{\pi}$ protons (total $N=N_{\nu}+N_{\pi}$ ) interacting via a one- plus two-body Hamiltonian, with the neutrons and protons each restricted to an active set of single-particle orbitals. For simplicity of presentation, we will focus in this section on just one type of particle, as is the case for the test applications we will report. In a later section, we will note those modifications required for neutron-proton systems.

We begin by splitting the set of multiply degenerate single-particle levels into an ordered set of doubly degenerate levels. This is most naturally done by carrying out an axially symmetric deformed Hartree-Fock (HF) calculation for the system. Each of the resulting HF levels is then doubly degenerate. Going from the set of spherical single-particle levels to the axially symmetric set of doubly degenerate levels is schematically illustrated in Fig. 1 for a problem involving eight identical nucleons in the $f-p$ shell.

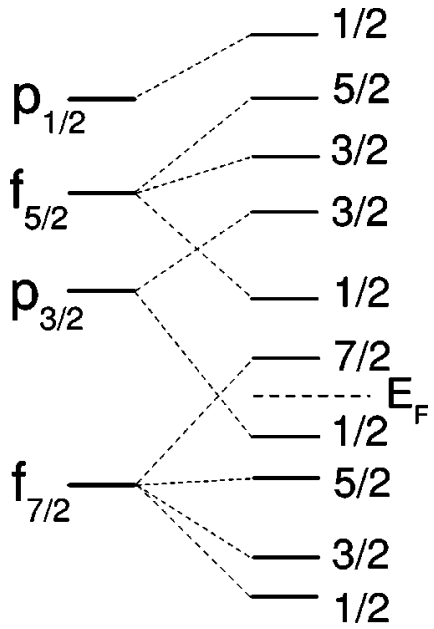

FIG. 1. Schematic illustration of the splitting of the model-space single-particle levels into a set of doubly degenerate levels by an axially deformed Hartree-Fock calculation. The dashed line represents the Fermi energy $\left(E_{F}\right)$, which separates the particle levels from the hole levels. Each doubly degenerate level is labeled by its angular momentum projection on the intrinsic $z$ axis.

Each of these doubly degenerate single-particle levels admits precisely four states. One is the state with no particles, one is the state with one particle having positive $m$ projection, one is the state with one particle having negative $m$ projection, and the last is the state containing two particles, with both positive and negative $m$.

This set of doubly degenerate single-particle levels naturally splits into two smaller sets, as likewise illustrated in Fig. 1. Those levels above the (noninteracting) Fermi surface are the particle levels and those below the Fermi surface are the hole levels. Reiterating what was stated earlier, we will systematically take into account all of the levels of the problem, by gradually moving away from the Fermi surface.

In this basis, the shell-model Hamiltonian takes the form

$$
\begin{aligned}
H= & \sum_{\alpha m} \epsilon_{\alpha m} a_{\alpha m}^{\dagger} a_{\alpha m} \\
& +\frac{1}{4} \sum_{\alpha_{1} m_{1} \alpha_{2} m_{2} \alpha_{3} m_{3} \alpha_{4} m_{4}}\left\langle\alpha_{1} m_{1}, \alpha_{2} m_{2}|V| \alpha_{3} m_{3}, \alpha_{4} m_{4}\right\rangle \\
& \times a_{\alpha_{1} m_{1}}^{\dagger} a_{\alpha_{2} m_{2}}^{\dagger} a_{\alpha_{4} m_{4}} a_{\alpha_{3} m_{3}}
\end{aligned}
$$

with both a single-particle energy term and a two-body interaction.

At this point, let us assume that we have already treated some of the levels of the problem, perhaps the first $n$ particle levels above the Fermi energy (forming a particle block) and the first $n$ hole levels below the Fermi energy (forming a hole block). Let us also assume that we have precisely $p$ particle states in the particle block and $p$ hole states in the hole block, i.e., the same number for each.

Furthermore, we will assume that we have calculated and stored the matrix elements of all possible sub-operators of the Hamiltonian within the $p$-dimensional particle and hole blocks, respectively. For a one- plus two-body Hamiltonian, 
the operators of relevance are $a^{\dagger}, a^{\dagger} a, a^{\dagger} a^{\dagger}, a^{\dagger} a^{\dagger} a$, and $a^{\dagger} a^{\dagger} a a$, as all others can be directly calculated from these using appropriate Hermitian adjoint relations.

We now enlarge both the particle and hole blocks, by adding one more (doubly degenerate) level to each. Clearly the number of states in the enlarged particle and hole blocks will be $4 p$. These states can be written as $|i, j\rangle=|i\rangle|j\rangle$, where $i=1, \ldots, p$ and $j=1, \ldots, 4$.

What we would like to do is to truncate both the particle and hole blocks from these $4 p$ states to the optimum $p$ states, precisely the same number that we had before adding the new level.

In order to accomplish this we first recalculate the matrix elements of all of the Hamiltonian sub-operators in the enlarged particle and hole blocks. To show how this is done, we will focus on one specific set of matrix elements, those of the operator $a_{\alpha_{1} m_{1}}^{\dagger} a_{\alpha_{2} m_{2}}$. Its matrix elements in the enlarged block can be readily expressed as

$$
\begin{aligned}
\left\langle i, j\left|a_{\alpha_{1} m_{1}}^{\dagger} a_{\alpha_{2} m_{2}}\right| k, l\right\rangle & \\
= & \left\langle i\left|a_{\alpha_{1} m_{1}}^{\dagger} a_{\alpha_{2} m_{2}}\right| k\right\rangle \delta_{j, l}+\left\langle j\left|a_{\alpha_{1} m_{1}}^{\dagger} a_{\alpha_{2} m_{2}}\right| l\right\rangle \delta_{i, k} \\
& +(-)^{n_{k}}\left\langle i\left|a_{\alpha_{1} m_{1}}^{\dagger}\right| k\right\rangle\left\langle j\left|a_{\alpha_{2} m_{2}}\right| l\right\rangle \\
& -(-)^{n_{k}}\left\langle i\left|a_{\alpha_{2} m_{2}}\right| k\right\rangle\left\langle j\left|a_{\alpha_{1} m_{1}}^{\dagger}\right| l\right\rangle,
\end{aligned}
$$

where $n_{i}$ is the number of particles in state $|i\rangle$. Depending on which of the two subspaces the operator indices refer (either the levels $1 \rightarrow n$ or the new level $n+1$ ), a different term in the sum applies. For example, if both $\alpha_{1}, m_{1}$ and $\alpha_{2}, m_{2}$ refer to single-particle states from the first $n$ levels, it is the first term that applies.

The key point is that all terms in Eq. (2) involve a product of a matrix element from the previous $p \times p$ space (levels 1 $\rightarrow n$ ) and a matrix element for the level that is being added. The matrix element from the previous space was already calculated and stored, whereas the one from the new level is very simple and can be written down analytically.

At this point we have calculated and stored all of the particle and hole matrix elements in their enlarged blocks. The next step is to construct the space of particle-hole states, by forming products of states in the particle and hole blocks, viz, $\left|i_{P}, j_{H}\right\rangle=\left|i_{P}\right\rangle\left|j_{H}\right\rangle$. This is usually referred to as the superblock.

We only build states in the superblock with the correct number of total particles $N$ for the problem of interest. That means that we only consider states with the same number of particles and holes. Furthermore, assuming that the Hamiltonian is rotationally invariant, it suffices to consider only those states for which the total $z$ projection of the angular momentum is zero.

Within this product space, we then build the Hamiltonian matrix. Using the simplified notation $\gamma=\{\alpha m\}$, this can be expressed as

$$
\begin{aligned}
\left\langle i_{P}, j_{H}\right| & H\left|k_{P}, l_{H}\right\rangle \\
= & \sum_{\gamma} \epsilon_{\gamma}\left[\left\langle i_{P}\left|a_{\gamma}^{\dagger} a_{\gamma}\right| k_{P}\right\rangle \delta_{j_{H}, l_{H}}+\left\langle j_{H}\left|a_{\gamma}^{\dagger} a_{\gamma}\right| l_{H}\right\rangle \delta_{i_{P}, k_{P}}\right] \\
& +\frac{1}{4} \sum_{\gamma_{i}, \gamma_{j}, \gamma_{k}, \gamma_{l}}\left\langle\gamma_{i}, \gamma_{j}|V| \gamma_{k}, \gamma_{l}\right\rangle \\
& \times\left[\delta_{i_{P}, k_{P}}\left\langle j_{H}\left|a_{\gamma_{i}}^{\dagger} a_{\gamma_{j}}^{\dagger} a_{\gamma_{l}} a_{\gamma_{k}}\right| l_{H}\right\rangle\right. \\
& +2(-)^{n_{k_{P}}}\left\langle i_{P}\left|a_{\gamma_{i}}^{\dagger}\right| k_{P}\right\rangle\left\langle j_{H}\left|a_{\gamma_{j}}^{\dagger} a_{\gamma_{l}} a_{\gamma_{k}}\right| l_{H}\right\rangle \\
& +2(-)^{n_{k_{P}}}\left\langle i_{P}\left|a_{\gamma_{l}}\right| k_{P}\right\rangle\left\langle j_{H}\left|a_{\gamma_{i}}^{\dagger} a_{\gamma_{j}}^{\dagger} a_{\gamma_{k}}\right| l_{H}\right\rangle \\
& +\left\langle i_{P}\left|a_{\gamma_{i}}^{\dagger} a_{\gamma_{j}}^{\dagger}\right| k_{P}\right\rangle\left\langle j_{H}\left|a_{\gamma_{l}} a_{\gamma_{k}}\right| l_{H}\right\rangle \\
& -4\left\langle i_{P}\left|a_{\gamma_{i}}^{\dagger} a_{\gamma_{l}}\right| k_{P}\right\rangle\left\langle j_{H}\left|a_{\gamma_{j}}^{\dagger} a_{\gamma_{k}}\right| l_{H}\right\rangle \\
& +\left\langle i_{P}\left|a_{\gamma_{l}} a_{\gamma_{k}}\right| k_{P}\right\rangle\left\langle j_{H}\left|a_{\gamma_{i}}^{\dagger} a_{\gamma_{j}}^{\dagger}\right| l_{H}\right\rangle \\
& +2(-)^{n_{k_{P}}}\left\langle i_{P}\left|a_{\gamma_{j}}^{\dagger} a a_{\gamma_{k}} a_{\gamma_{l}}\right| k_{P}\right\rangle\left\langle j_{H}\left|a_{\gamma_{i}}^{\dagger}\right| l_{H}\right\rangle \\
& +2(-)^{n_{k_{P}}}\left\langle i_{P}\left|a_{\gamma_{i}}^{\dagger} a_{\gamma_{j}}^{\dagger} a_{\gamma_{l}}\right| k_{P}\right\rangle\left\langle j_{H}\left|a_{\gamma_{k}}\right| l_{H}\right\rangle \\
& \left.\times \delta_{j_{H}, l_{H}}\left\langle i_{P}\left|a_{\gamma_{i}}^{\dagger} a_{\gamma_{j}}^{\dagger} a_{\gamma_{l}} a_{\gamma_{k}}\right| k_{H}\right\rangle\right] .
\end{aligned}
$$

Note that every term in Eq. (3) involves a product of a matrix element in the particle space and a matrix element of the conjugate operator in the hole space. All of these matrix elements, for both particles and holes, have already been calculated and stored in their respective enlarged blocks.

Next we diagonalize the superblock Hamiltonian, viz.,

$$
H\left|\Psi_{k}\right\rangle=E_{k}\left|\Psi_{k}\right\rangle,
$$

with

$$
\left|\Psi_{k}\right\rangle=\sum_{i_{P}, j_{P}=1,4 p} \Psi_{i j}^{(k)}\left|i_{P}\right\rangle\left|j_{H}\right\rangle
$$

Note that both sums go over the $4 p$ states in the respective enlarged particle and hole blocks.

We now wish to construct the optimal approximation to the ground state of the system (or to some low-lying set of states) that is achieved when we only retain $p$ particle states and $p$ hole states. By optimal, we will mean the approximation that maximizes the overlap between the truncated state and the exact ground state of the superblock $\left[\left|\Psi_{1}\right\rangle\right.$ in Eq. (5)]. For now, we will ask for the optimal description of the ground state; later we will discuss how to generalize this to several states.

We will perform the optimized truncation in two stages, first asking what is the optimal approximation when we truncate the particle states and then asking what is the optimal approximation when we truncate the hole states. For simplicity of notation, we will let $|\Psi\rangle=\left|\Psi_{1}\right\rangle$ and $\Psi_{i j}=\Psi_{i j}^{(1)}$.

Let us focus first on the particle block. We wish to find a subset of particle states ( $p$ in number), such that the truncated ground state built up from these states has the largest 
possible overlap with the exact superblock ground state. To accomplish this, we first define the reduced density matrix for particles,

$$
\rho_{i_{P} i_{P}^{\prime}}^{P}=\sum_{j_{H}=1,4 p} \Psi_{i_{P} j_{H}} \Psi_{i_{P}^{\prime} j_{H}}^{*} .
$$

The reduced density matrix separates into blocks according to the number of particles and the $m$ value.

We then diagonalize this $4 p \times 4 p$ matrix,

$$
\rho^{P}\left|u_{P}^{\alpha}\right\rangle=\omega_{\alpha}^{P}\left|u_{P}^{\alpha}\right\rangle .
$$

A given eigenvalue $\omega_{\alpha}^{P}$ represents the probability of finding the particle state $\left|u_{P}^{\alpha}\right\rangle$ in the full ground state wave function $|\Psi\rangle$. Thus, the optimal truncation in the sense described above corresponds to retaining the $p$ eigenvectors $\left|u_{P}^{\alpha}\right\rangle$ that have the largest eigenvalues.

We then do exactly the same for the hole block. Namely, we construct the reduced density matrix for holes,

$$
\rho_{j_{H} j_{H}^{\prime}}^{H}=\sum_{i_{P}=1,4 p} \Psi_{i_{P} j_{H}} \Psi_{i_{P} j_{H}^{\prime}}^{*},
$$

diagonalize it, and then keep only the $p$ hole states with the largest eigenvalues.

At this point, we have identified the $p$ particle states and $p$ hole states that best approximate the full ground state of the coupled superblock. The final step before proceeding to the next level is to transform all of the matrix elements that we calculated in the $4 p$-dimensional particle and hole spaces to the optimal $p$-dimensional truncated spaces.

As noted earlier, we need not target our optimization to the ground state only. We can target it to any set of states we wish. If, for example, we wanted to optimally describe the lowest $L$ eigenstates of the system, we would build mixed density matrices

$$
\begin{gathered}
\rho_{i_{P} i_{P}^{\prime}}^{P}=\frac{1}{L} \sum_{k=1, L} \sum_{j_{H}} \Psi_{i_{P} j_{H}}^{(k) *} \Psi_{i_{P}^{\prime} j_{H}}^{(k)}, \\
\rho_{j_{H} j_{H}^{\prime}}^{H}=\frac{1}{L} \sum_{k=1, L} \sum_{i_{P}} \Psi_{i_{P} j_{H}}^{(k) *} \Psi_{i_{P} j_{H}^{\prime}}^{(k)},
\end{gathered}
$$

and use them to choose the $p$ most important particle and hole basis states to retain.

Once this series of steps has been implemented, we simply return to the point where the discussion began and continue iteratively. Namely, we add the next levels, precisely as we did above, first calculating and storing all matrix elements in the enlarged particle and hole blocks, then constructing and diagonalizing the Hamiltonian matrix in the superblock, then constructing and diagonalizing the reduced density matrices for particles and holes (depending on the states we wish to target) and then truncating to the most important $p$ states for particles and holes, respectively, based on the eigenvalues of the associated density matrices.

Were the number of particle and hole levels the same, we would simply proceed as above to treat all levels. We would initialize the iteration process by treating the two levels nearest to the Fermi surface, whose matrix elements can be calculated very simply. We would then continue to add levels as defined above. Truncation would not be necessary until the number of particle/hole states exceeds $p$. Prior to that point, it is not necessary to construct the Hamiltonian matrix in the superblock, since it is only needed for the purpose of truncation. Note that in the absence of truncation, the number of particle and hole states after treating $n$ levels is $4^{n}$. Thus, until $4^{n}>p$, truncation is not required.

When there are different numbers of particle levels and hole levels, some modification to the above algorithm is needed. Assume, for example, that there are more particle levels than hole levels, so that the system is less than half full. We can continue to add two levels at a time, one for particles and one for holes, until we have exhausted all of the hole levels. From that point on, however, there are no hole levels to add. Thus, in subsequent iterations, we only add particle levels and only carry out the optimized truncation for those states.

At each iteration, there are in fact $4 p$ states for particles and holes in the enlarged blocks required to build the superblock Hamiltonian. It is only after truncation that it is reduced to $p$. Assuming that there are more particle levels than hole levels, we have a choice of how to proceed after the hole levels have been exhausted. One possibility is not to carry out a truncation of the hole levels at its last iteration, keeping $4 p$ hole states in subsequent iterations. The other is to carry out a truncation at the last hole iteration and then keep only $p$ hole states subsequently. The former is more accurate; the latter reduces (perhaps significantly) the storage needs for the calculation. Considering that storage issues are likely to be especially important for subsequent applications of the methodology to more complex systems, it is useful to assess the accuracy lost if we keep only $p$ hole states after the hole levels have been exhausted. This is one of the issues we will address in the test applications to follow.

\section{Neutrons and protons}

In the presence of both neutrons and protons, the same formalism applies, but with a few minor practical modifications.

(a) In a system of neutrons and protons, there are four distinct blocks-neutron particle, proton particle, neutron hole, and proton hole.

(b) In a given iteration, we have a choice of how many and which levels to include. One possibility is to add a particle level and a hole level of the same type, switching between proton levels and neutron levels from one iteration to the next. The other possibility is to add four levels in an iteration-one for each of the four blocks. Both options can readily be implemented with the formalism of the preceding section and both permit us to eventually include all active levels. We expect that the first approach, in which we add a particle and a hole level in each iteration, should suffice for heavy nuclei where the active neutrons and protons occupy different major shells. For lighter nuclei where they occupy the same major shells, it is most likely preferable to add all four levels at the same time. 
(c) The superblock basis in which the Hamiltonian is diagonalized consists of products of states of all four typesneutron particle, neutron hole, proton particle, and proton hole. Only those states with the correct number of neutrons and protons and with total angular momentum projection zero need be considered.

(d) The reduced density matrix for a given block requires a contraction over the states of the other three blocks.

(e) We need to consider Hamiltonians that include singleparticle energy terms for neutrons and protons as well as the neutron-neutron, proton-proton, and neutron-proton interactions. The matrix elements for all of these terms can be readily obtained from the same basic like-particle operators as before $\left(a^{\dagger}, a^{\dagger} a, a^{\dagger} a^{\dagger}, a^{\dagger} a^{\dagger} a\right.$ and $\left.a^{\dagger} a^{\dagger} a a\right)$.

\section{THE MODEL}

The first application of the $p-h$ DMRG, as described in the preceding section, was to a problem of a very large number of particles interacting via a pairing force and constrained to a set of equally spaced doubly degenerate singleparticle levels [8]. This problem, which is of direct relevance to the physics of ultrasmall superconducting grains, was solved at half-filling for up to 400 levels. Despite the enormity of the model space, especially for a very large number of levels, the DMRG method was able to reproduce the ground state of the system very accurately, which could be obtained exactly using a method pioneered by Richardson [10]. These results suggest that the $p-h$ DMRG method can very accurately describe the properties of fermion systems that are dominated by a single collective degree of freedom.

Nuclei, on the other hand, are characterized by several competing collective degrees of freedom, most importantly those associated with the pairing and quadrupole fields. Thus, before applying the DMRG method with confidence to nuclear systems, it is necessary to first demonstrate that it continues to work well in the presence of such competing collective effects.

With that in mind, we report here a series of test calculations for systems that admit pairing plus quadrupole correlations on the same footing. The simplest such system is one involving identical nucleons restricted to a single level with large degeneracy and interacting via a pairing plus quadruple force. Unfortunately, the $\left(0^{+}\right)$ground state for an even number of particles occupying a single level invariably has equal population of all $m$ substates and thus such a problem does not admit a Fermi surface. Since a Fermi surface is an important characteristic of real Fermi systems, including nuclei, it is important to permit one in the test calculations. Thus, in the calculations to follow we supplement the pairing plus quadrupole interaction of our test model with a singleparticle energy term that splits the multiply degenerate large$j$ orbit into a series of doubly degenerate levels. The Hamiltonian we use takes the form

$$
H=-\chi Q \cdot Q-g P^{\dagger} P-\epsilon \sum_{m}|m| a_{j m}^{\dagger} a_{j m} .
$$

The last term splits the levels of the single- $j$ shell into a set of equally spaced levels of "oblate" character, with the largest $|m|$ value lowest. Because of the last term, the Hamiltonian is not in general rotationally invariant and thus its eigenstates do not have conserved angular momentum. Rotational invariance is, of course, recovered for $\epsilon=0$.

In the calculations that we will describe, we use a scaled version of the quadrupole-quadrupole strength $\chi$, defined according to

$$
\chi=\frac{\langle j\|Q\| j\rangle^{2}}{2 j+1} \tilde{\chi}
$$

where $\tilde{\chi}$ is the usual $Q \cdot Q$ coupling strength. With this definition, $\chi$ and $g$ have the same dimensions, which we will subsequently ignore.

\section{RESULTS FOR TEN PARTICLES IN A $j=25 / 2$ ORBIT}

\section{A. General remarks}

In this section, we report the results of calculations for a system of ten identical particles in a $j=25 / 2$ orbit. In this case, there are 13 active doubly degenerate orbits, five for holes and eight for particles. The Fermi surface lies between the $m=17 / 2$ hole level and the $m=15 / 2$ particle level. For this problem, the size of the space that would be required for exact diagonalization (assuming that we only consider $m$ $=0$ states) is 109583 , well within the limits of standard diagonalization routines. Thus, for this set of calculations, we can readily compare the results of calculations based on the DMRG methodology with the corresponding exact results, and thereby obtain important insight into the usefulness of the method. We will first report calculations for the standard scenario, in which (i) we only target the ground state in the DMRG truncation strategy and (ii) we include the full complement of $4 p$ hole states in the hole block after all hole levels have been treated. We will then present results for scenarios that systematically modify these two assumptions.

\section{B. Results for standard scenario}

Here we present results in which we only target the ground state and we include all $4 p$ hole states in the hole block after the hole levels have been exhausted.

In our earlier paper, we presented results for two cases, one with $\chi=1, g=0, \epsilon=0.1$ and one with $\chi=1, g$ $=0.05, \epsilon=0.1$. In both cases, we achieved accuracy for the ground state energy of roughly 1 part in $10^{6}$ with reasonable values of $p$.

Obviously the case with $g=0$ involves pure quadrupole correlations. On the basis of Hartree-Fock-Bogoliubov (HFB) calculations that we have now performed, we conclude that the $g=0.05$ case likewise is dominated by quadrupole correlations, with pairing correlations minimal. Since it is our desire here to assess the DMRG method in the typical nuclear scenario in which different correlation effects compete, we will show throughout this section results for a further-enhanced pairing interaction, namely, for $\chi=1, g$ $=0.1, \epsilon=0.1$. In this case, the HFB calculations show a well developed superconducting solution. This can be readily 


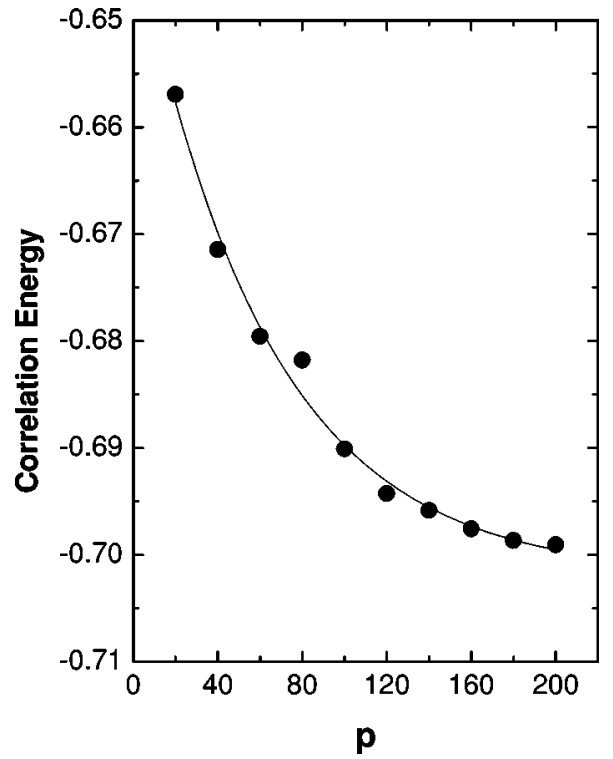

FIG. 2. DMRG correlation energies as a function of $p$ for a system of ten particles in a $j=25 / 2$ orbit subject to a Hamiltonian with $\chi=1, g=0.1$, and $\epsilon=0.1$. The solid line represents an exponential fit to the DMRG results.

seen by noting that for this problem the ratio of the average pairing gap to the mean spacing between HF levels is $0.39 / 0.18=2.1$.

Figure 2 shows results for the DMRG correlation energies, defined as the gain in energy relative to Hartree-Fock approximation. The exact and HFB values are

$$
E_{\text {corr }}^{\text {Exact }}=-0.70633 ; \quad E_{\text {corr }}^{H F B}=-0.20641 .
$$

By a $p$ value of 200 , we obtain more than $99 \%$ of the exact correlation energy, a dramatic improvement over HFB approximation, which only gives $28 \%$.

For $p=200$ the largest superblock matrix that had to be diagonalized had a dimension of 2886. For $p=40$, the maximum dimension was 232; nevertheless, for this value of $p$ we still achieve over $95 \%$ of the full correlation energy.

Table I shows results for the excitation energies of the lowest states of this same system. Despite the fact that we only targeted the ground state in the density matrix phase of these calculations, the agreement is as good for the excitation

TABLE I. Excitation energies for ten particles in a $j=25 / 2$ level. The Hamiltonian parameters are $\chi=1, g=0.1$, and $\epsilon=0.1$.

\begin{tabular}{rccc}
\hline \hline$p$ & $E_{1}$ & $E_{2}$ & $E_{3}$ \\
\hline 40 & 0.89040 & 1.26036 & 1.49354 \\
80 & 0.88266 & 1.18608 & 1.41182 \\
120 & 0.88791 & 1.18077 & 1.41795 \\
160 & 0.88794 & 1.17722 & 1.41456 \\
200 & 0.88784 & 1.17302 & 1.41024 \\
\hline Exact & 0.88578 & 1.16245 & 1.39915 \\
\hline \hline
\end{tabular}

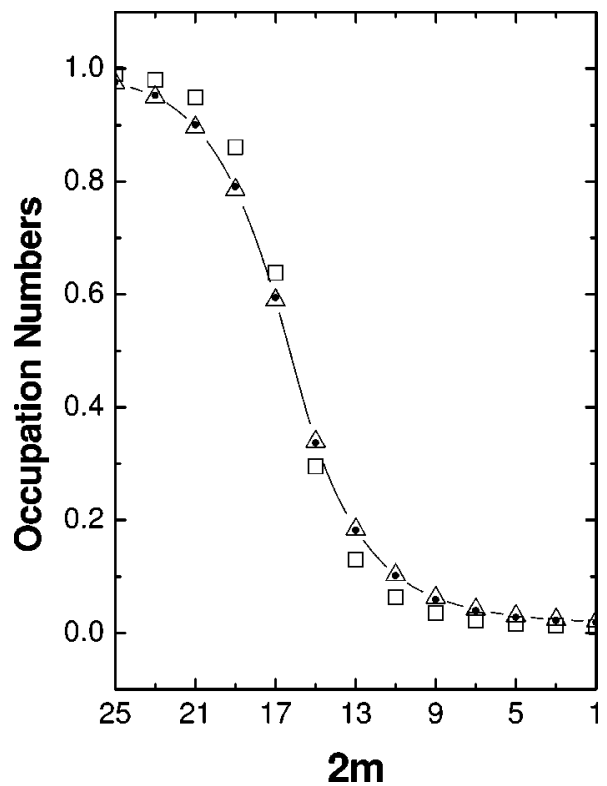

FIG. 3. The occupation numbers for the same system as in Fig. 2. The horizontal label $2 m$ is twice the $z$ projection of the angular momentum. The solid circles (connected to guide the eye by the solid line) represent the exact results; the open diamonds represent the DMRG results for $p=200$; the open squares represent the HFB results.

energies of these low-lying states as it is for the ground state energy. By $p=200$, all three excitation energies are reproduced to $1 \%$ or better.

In Fig. 3, we show the exact, DMRG and HFB results for the occupation numbers of the particle and hole levels associated with the ground state solution. The DMRG results, which are shown for $p=200$, are in excellent agreement with the exact results for all levels with appreciable occupation. In contrast, the HFB calculation does not obtain sufficient depletion of the Fermi sea.

From these results, we conclude that the $p-h$ DMRG method is able to describe with extreme accuracy the lowlying properties of complex many-body systems with competing collective features.

\section{Dependence on the number of states targeted}

What is the importance of including excited states in the targeting procedure? We address this in Fig. 4, where we compare the results for the ground state energy and the energies of low-lying excited states when different sets of states are targeted. The solid triangles refer to results when only the ground state is targeted $(L=1)$. The open circles are the results obtained when the lowest four states are all targeted in the optimization procedure $(L=4)$. We also include in the figure the exact results.

The results are not unexpected. The ground state is better described when it is the only state targeted in the optimization procedure. Furthermore, in such a scenario a reasonable description of the low-lying excited states is achieved. When several states are targeted, the agreement gets worse, albeit only marginally, for the ground state, but becomes better for 


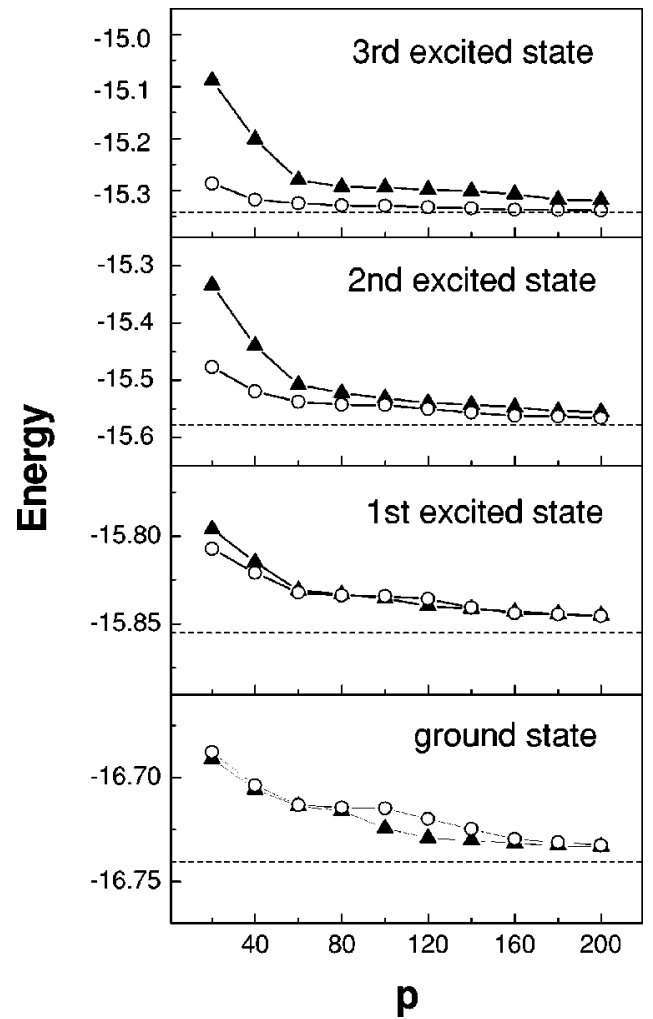

FIG. 4. The energy of the ground state and of the three lowest excited states for the same system as in Fig. 2. The solid triangles are the results when only the ground state is targeted in the optimization procedure; the open circles refer to calculations when the lowest four states of the system are targeted simultaneously. The exact results are represented by dashed lines.

the excited states. What is most important, however, is that once $p$ becomes large enough, there is no discernable difference between the two sets of results, both of which are also in excellent agreement with the exact eigenenergies.

\section{Dependence on how we treat the hole block after all hole levels have been exhausted}

Next we address the effect of maintaining all $4 p$ hole states following treatment of the last hole level. This was addressed by carrying out the DMRG calculations in two ways, namely, keeping only $p$ hole states after the last hole iteration or keeping $4 p$. For a value of $p=200$, we find for the ground state energy a value of -16.7331 when only $p$ states are retained and a value of -16.7333 when all $4 p$ are kept. The gain in energy through the improved treatment of the hole states is just 1 part in $10^{6}$. On the basis of these results, the subsequent calculations we describe will be based on the simplifying, but highly justified, assumption that we maintain just $p$ hole states in those iterations that only add particle levels.

\section{RESULTS FOR 40 PARTICLES IN A $j=99 / 2$ ORBIT}

The excellent quality of the results obtained in the test calculations of the preceding section has encouraged us to treat a much larger system, one for which exact diagonaliza-

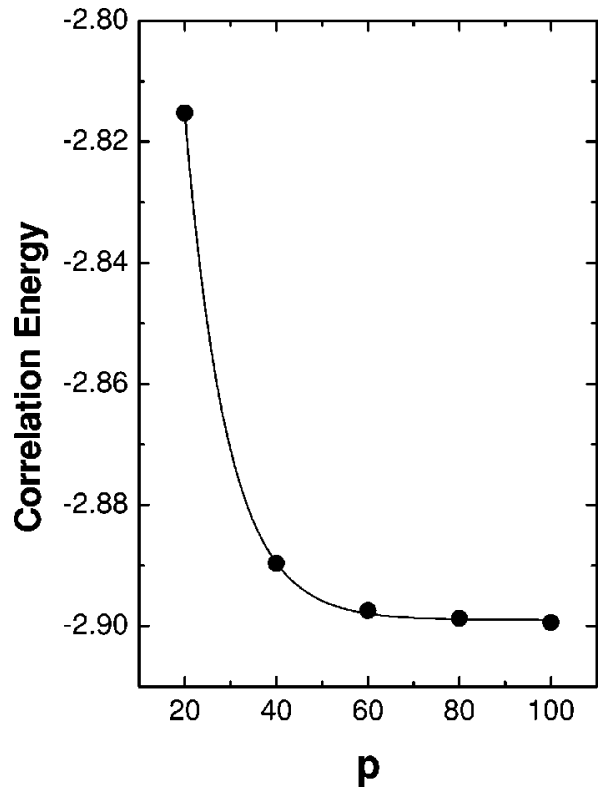

FIG. 5. The ground-state energy for a system of 40 identical nucleons in a $j=99 / 2$ orbit with the Hamiltonian parameters $\chi$ $=1, g=0.1$, and $\epsilon=0.2$. An exponential fit to these results is also plotted.

tion is not possible. Following an optimization of the DMRG calculational methodology, we are now able to treat systems significantly larger than were feasible in Ref. [9]. Here we report the largest calculation we have so far carried out-for a system of 40 particles occupying a $j=99 / 2$ orbit. In this case, the exact calculation would involve a Hamiltonian matrix of dimension $3.84 \times 10^{25}$, obviously much too large to treat without dramatic truncation. The parameters of the Hamiltonian of Eq. (10) that were used in this calculation are $\chi=1, g=0.1$, and $\epsilon=0.2$. The calculations targeted only the ground state and retained only $p$ hole states after treating the 20 hole levels. (The latter is especially important when treating extremely large problems such as this one.) The results for the ground state correlation energy are shown in Fig. 5. By $p=100$, we obtain a ground state correlation energy of - 2.8994. An exponential fit to the DMRG results, likewise indicated in the figure, gives an asymptotic result for the ground state energy of $-2.8990 \pm 0.0003$. This is close enough to our DMRG result with $p=100$ to suggest that we have achieved accuracy for the ground state correlation energy to 1 part in $10^{4}$.

We have also carried out an HFB calculation for this system, which confirms that the ground state is superconducting. The correlation energy achieved in this calculation is -1.7902 , less than $62 \%$ of that estimated from the exponential fit to the DMRG results.

\section{CONCLUSION}

In this paper, we describe the recently developed particlehole density matrix renormalization group method and report its test application to a problem involving many identical nucleons constrained to a single- $j$ shell and subject to a Hamiltonian with a pairing plus quadrupole interaction and a 
single-particle term that splits the shell into doublets. Earlier work along these lines was reported in Ref. [9]. Here we develop the formalism in greater detail and report calculations that address some important aspects of the method that needed to be clarified before the method could be meaningfully applied to more realistic nuclear systems.

In our view, the current calculations confirm and expand on the conclusions reported in Ref. [9] regarding the use of the method in large-scale shell-model calculations. As long as there is a well defined Fermi surface in the problem, the method is able to produce extremely accurate results for the ground state energy of the system and for the energies of low-lying excited states as well, even in the presence of competing collective correlation effects. It is also able to accurately reproduce other (number-conserving) properties of the system, including, for example, occupation numbers. Based on an optimized computational methodology, we are now able to treat much larger problems than in Ref. [9], and this bodes well for subsequent more-realistic applications of the methodology. In all cases we have studied, even those very large-scale problems we are now able to handle only because of the optimized methodology, we seem to be able to obtain accurate results while diagonalizing matrices of moderate di- mensions. Critical to the success of the method is the rapid exponential convergence that it typically exhibits as a function of the number of particle and hole states maintained in a given calculation.

Clearly the next step is for us to further develop the method for use in realistic nuclear systems. This will mean including both neutrons and protons, several nongenerate single-particle levels, and general nuclear Hamiltonians. The framework for these extensions is spelled out in Sec. II and is currently in the process of being implemented.

\section{ACKNOWLEDGMENTS}

This work was supported in part by the National Science Foundation under Grant No. PHY-9970749, by the Spanish DGI under Grant No. BFM2000-1320-C02-02, by NATO under Grant No. PST.CLG.977000, by the Bulgarian Science Foundation under Contract No. $\Phi-905$, and by the Bulgarian-Spanish Exchange Program under Grant No. 2001BG0009. One of the authors (S.S.D.) would also like to acknowledge the partial support of a Fulbright Visiting Scholar Grant.
[1] See, e.g., K. Heyde, The Nuclear Shell Model (SpringerVerlag, Berlin, 1990).

[2] E. Caurier et al., Phys. Rev. C 59, 2033 (1999).

[3] M. Honma, T. Mizusaki, and T. Otsuka, Phys. Rev. Lett. 77, 3315 (1996).

[4] M. Shimizu, T. Otsuka, T. Mizusaki, and M. Honma, Phys. Rev. Lett. 86, 1171 (2001).

[5] S.R. White, Phys. Rev. Lett. 69, 2863 (1992); Phys. Rev. B 48,
10345 (1993).

[6] S.R. White, Phys. Rep. 301, 187 (1998).

[7] J. Dukelsky and G.G. Dussel, Phys. Rev. C 59, R3005 (1999).

[8] J. Dukelsky and G. Sierra, Phys. Rev. Lett. 83, 172 (1999); Phys. Rev. B 61, 12302 (2000).

[9] J. Dukelsky and S. Pittel, Phys. Rev. C 63, 061303(R) (2001).

[10] R.W. Richardson, Phys. Rev. 141, 949 (1966). 